\title{
La ética del cuidado en la Pedagogía Saludable
}

\section{The Ethics of Care in Healthy Pedagogy}

\author{
Ileana Castillo Cedeño ${ }^{1}$ \\ Universidad Nacional \\ Centro de Investigación y Docencia en Educación \\ Heredia, Costa Rica \\ ileana.castillo.cedeno@una.cr \\ Rocío Castillo Cedeño ${ }^{2}$ \\ Universidad Nacional \\ Centro de Investigación y Docencia en Educación \\ División de Educación Básica \\ Heredia, Costa Rica \\ rociocastillocedeno@gmail.com
}

Luz Emilia Flores Davis ${ }^{3}$

Universidad Nacional

Centro de Investigación y Docencia en Educación

División de Educación Básica

Heredia, Costa Rica

luz.flores.davis@una.cr

Giselle Miranda Cervantes ${ }^{4}$

Universidad Nacional

Centro de Investigación y Docencia en Educación

División de Educación Básica

Heredia, Costa Rica

giselle.miranda.cervantes@una.cr

Recibido: 15 noviembre 2013 Aceptado: 4 diciembre $2014 \quad$ Corregido: 3 octubre 2014

1 Doctora en Educación con especialidad en Mediación Pedagógica. Egresada del Doctorado en Diseño Curricular y Evaluación Educativa, de la Universidad de Valladolid, España. Magíster en Psicopedagogía. Magíster en Educación Preescolar. Especialista en didáctica de la educación del consumidor. Universidad de Valladolid, España. Profesora, bachiller y licenciada en ciencias de la Educación con énfasis en educación preescolar, Universidad Nacional de Costa Rica. Actualmente se desempeña como investigadora y ocupa el cargo de Decana del Centro de Investigación y Docencia en Educación de la Universidad Nacional de Costa Rica. Autora de varias publicaciones sobre educación, pedagogía, diversidad, liderazgo, educación superior en revistas de su especialidad.

2 Doctora en Educación con mención en Mediación Pedagógica. Magíster en Administración Educativa y en Psicopedagogía. Profesora y Bachiller en ciencias de la Educación con énfasis en educación preescolar, Universidad Nacional. Ha participado activamente en procesos de formación de formadores, principalmente, en el diseño e implementación de cursos de pedagogía a distancia, en la Universidad Estatal a Distancia. Durante cinco años se desempeñó como decana de la Facultad de Educación de la Universidad De La Salle Costa Rica y trabajó durante un año como Vicerrectora Académica en esta misma universidad. Actualmente, se desempeña como docente e investigadora en el Centro de Investigación y Docencia en Educación de la Universidad Nacional de Costa Rica. Autora del libro: La conciencia cósmica en la primera infancia. Reflexiones paradigmáticas, propuestas biopedagógicas y un acercamiento a la espiritualidad de las niñas y los niños.

3 Doctora en Educación con especialidad en Mediación Pedagógica. Máster en Educación Superior. Catedrática universitaria. Ha ocupado los cargos de Directora de Docencia, Directora de Desarrollo Profesional y Cooperación Universitaria, Presidenta de la Junta de Becas y Coordinadora de la Comisión Técnica de Admisión de la Universidad Nacional. Actualmente, se desempeña como investigadora, extensionista y docente en la División de Educación Básica del Centro de Investigación y Docencia en Educación (CIDE) de la Universidad Nacional, Costa Rica. Entre sus publicaciones más recientes destacan trabajos para la alfabetización de personas adultas.

4 Doctora en Educación con especialidad en Mediación Pedagógica. Bachiller, Licenciada y Máster en Psicología de la Universidad de Costa Rica. Labora como académica e investigadora desde 1990 en la División de Educación Básica del Centro de Investigación y Docencia en Educación (CIDE) de la Universidad Nacional de Costa Rica, unidad académica en la que ocupó los cargos de Directora, miembro del Consejo Académico y Coordinadora de las carreras de Educación Especial y de Enseñanza del Inglés para I y II ciclos. Miembro del Consejo Editorial de la Revista EDUCARE. Su interés en el campo de la investigación destaca las áreas de diversidad, seguimiento a egresadas de la carrera de educación especial, relaciones interpersonales, clima social de aula y pedagogía saludable. 
Resumen: En el marco del proyecto de investigación: Pedagogía Saludable que desarrolla la División de Educación Básica, del Centro de Investigación y Docencia en Educación (CIDE) de la Universidad Nacional de Costa Rica (UNA); este ensayo tiene el objetivo de proponer una vinculación entre pedagogía y salud, a la luz de una visión ética del cuidado. Las autoras proponen así, la Pedagogía saludable como un nuevo nodo y asumen el concepto de convivencialidad, entendiendo que todo ser vivo es fundamental en el tejido de la trama de la vida. El paradigma del cuidado es abordado como la relación amorosa consigo mismo, con el otro, con la otra y con todo lo existente frente a la situación crítica que vive la humanidad y la Madre Tierra, es decir, como una alternativa a la agresión y a la destrucción que amenaza no solo al planeta y a todos los seres vivientes, sino a las interrelaciones más elementales. Es así como las autoras, concluyen que la ética del cuidado significa un compromiso en la impostergable construcción de un mundo enlazado en y por el afecto, que abarque personas portadoras de los más altos valores y garantice la continuidad y el bienestar de todas las formas de vida.

Palabras claves: Cuidado, ética, comunidades de aprendizaje, convivencia, pedagogía saludable.

Abstract: In the scope of the Healthy Pedagogy Investigation Project, currently under development at the Elementary Education Division of the Costa Rican National University's (UNA) Center for Investigation and Teaching in Education (CIDE), this essay suggests a relationship between pedagogy and health, based on an ethical vision of caring. The authors propose the understanding of Healthy Pedagogy as a new node, which includes the concept of cohabitation and considers that all living creatures are fundamental for the fabric of life. The paradigm of caring is approached as a love relationship with ourselves, with others, and with all existence, facing the critical situation that both humanity and Mother Earth currently have, as an alternative to the aggression and destruction that threatens not only the planet and all living creatures, but the most basic interrelationships. From this perspective, the authors conclude that the ethics of caring represent a commitment towards the urgent construction of a world held together by affection, populated by people with the highest principles and which may guarantee the continuity and wellbeing of all living forms.

Key words: Caring, ethics, learning community, coexistence, healthy pedagogy.

\section{El cuidado como desafío pedagógico}

Hablar del ser humano y de las relaciones que este establece con las demás personas, consigo mismo y con el cosmos del que forma parte, implica abordar también el vínculo que determina la calidad de dichas relaciones. Esto, desde una perspectiva holista, está inmerso en un mar relacional de cuidado, afecto y sensibilidad que involucra la sabiduría, esa capacidad interior capaz de ensanchar los corazones y transformar nuestra miradas en un acto de amor, de fe y compromiso con nuestro diario vivir, con la existencia y sus interconexiones.

Las relaciones humanas, por su misma naturaleza, son complejas, de ahí que, desde la propuesta de una pedagogía saludable, se requiere no solo abordar la complejidad de los procesos de aprendizaje, sino comprenderlos y asumirlos como procesos vitales. La complejidad implica:

Forjar un marco conceptual completamente distinto que permita concebir los sistemas multidimensionales nacidos en una dinámica relacional y que nos llevan a pensar(nos) y a construir mundos de sentido muy diferentes a los que surgen de los modelos de pensamiento basados en oposiciones binarias. (Najmanovich, 2001, p. 2) 
La autora citada, claramente distingue entre: un pensamiento mecanicista a la luz del cual se establecen relaciones caracterizadas en la diferenciación sujeto-objeto fundamentadas en el principio de simplicidad y en la disección de un cuerpo-máquina, abstracto, separado de toda emotividad, conocimiento del otro, de la otra y del medio ambiente; y un pensamiento holista que aborde la salud como "una problemática del vivir humano como 'sujetos entramados' (...) para entrar al espacio cognitivo de la dinámica vincular, de los procesos y de las redes" (Najmanovich, 2001, p. 6).

Según lo indica Dossey (1999), todo lo existente baila al ritmo de una "biodanza" silenciosa que nos mantiene en equilibrio permanente con la Tierra y no hay ser alguno que se encuentre estrictamente demarcado; por eso "Nuestras raíces son profundas; estamos anclados en las estrellas" (p. 124). Es precisamente esta perspectiva la que nos lleva a cuestionarnos que, de continuar la humanidad inmersa en una sociedad que aún mantiene un esquema claramente divorciado de esta integralidad e interconexión, se priva de sentido a la comunidad planetaria de la que se forma parte, como esas fibras sensibles de ese hermoso tejido relacional que involucra la vida.

A partir de lo anterior, este ensayo tiene el objetivo de asumir el desafío de relacionar pedagogía y salud, lo cual implica construir nuevas visiones críticas del conocimiento que, fundadas en una comprensión compleja del pensamiento y la vida, posibiliten restituir la vitalidad de la existencia a partir de modelos de autoorganización (Maturana y Varela, 1986) desde los cuales, la salud sea pensada "en referencia al itinerario que la misma vida fija, a los valores que el hombre construye, a las prácticas culturales que le dan sentido" (Najmanovich, 2001, p. 7).

Desde esta perspectiva compleja, reviste particular importancia el cuidado dado que, “(...) la pedagogía es un proceso en el cual las experiencias de vida tienen un papel trascendental y son influencias por el amor; en este sentido, la pedagogía permite visualizar los procesos desde la hermandad, la bondad, el encuentro genuino y el cuidado" (Castillo, Castillo, Flores y Miranda, 2014, p. 315).

\section{El cuidado, fibra sensible y ética que hilvana la pedagogía saludable}

De acuerdo con lo planteado por Boff (2012b):

Cuidado es una constante cosmológica. Si las energías originarias y los elementos y los primeros elementos no estuviesen regidos por un cuidado solidario para que todo mantuviese su debida proporción, el universo no habría surgido y nosotros no estaremos aquí escribiendo sobre el cuidado. Nosotros mismos somos hijos e hijas del cuidado. Si nuestras madres no nos hubiesen acogido con infinito cuidado, no habríamos tenido cómo bajar de la cuna e ir a buscar nuestro alimento. El cuidado es la condición previa que permite que un ser venga a la existencia. (p. 1)

El cuidado como imperativo ético enfatiza en la urgencia de orientar nuestras acciones para proteger el todo, pasar de un antropocentrismo devorador a una posición de cuidado profundo, dado que lo que actualmente está en debate, es la vida. La mayoría de tendencias, 
incluso algunas más conservadoras, admiten que la vida está amenazada, por ello se requiere, de una religación con el prójimo, con una comunidad, con una sociedad, con el cosmos. "Hay que construir un nuevo "ethos" que permitan una nueva convivencia entre los seres humanos y los demás seres de la comunidad biótica, planetaria y cósmica, que propicie nuevamente la fascinación ante la majestad del universo y la complejidad de las relaciones" (Boff, 2002, p. 26).

La ética del cuidado está estrechamente ligada con la posibilidad genuina del encuentro y la comprensión de que estamos en una trama de interrelaciones, que nuestros actos afectan no solo nuestra realidad cercana, sino también aquella lejana, nuestra influencia, por pequeña que sea, es poderosa. Una acción no solo debe mirar por el bienestar de los otros para el futuro, sino que debe basarse en la autenticidad del momento, ser verdadera en sí misma y ejercitar los valores de la compasión, el amor y la amabilidad, condiciones básicas para una ética del cuidado.

Experimentar la solidaridad con todo el universo tiene mucho que ver con el hecho de liberarnos a nosotros mismos y a nosotras mismas del hábito crónico de pensar que somos meros fragmentos inconexos, dejar de poner el énfasis en el yo aislado y en la conciencia de que nosotros o nosotras solo podemos conocer individualmente para pasar a considerar que somos capaces de pensar de manera conjunta.

El cuidado es un principio ético de la pedagogía y va de la mano con la necesidad de una humildad básica que permita reconocer que la clave para el cambio no radica tanto en una acción individual como en el modo en que interactuamos. Cuando se trabaja con afecto y simpatía, se pueden producir grandes transformaciones para una vida saludable.

Boff (2012b) señala cuatro significados del cuidado:

El primero indica que el cuidado es una actitud que implica amor, una relación de amistad con armonía y protección. El segundo significado establece que el cuidado es toda clase de preocupación e inquietud por personas y realidades con las cuales estamos implicados de forma afectiva. Como tercero, considera la necesidad y voluntad de proteger y ser protegido o protegida y, como cuarto y último significado, señala la importancia de la precaución que se ve reflejada en aquellas acciones o actitudes que es necesario evitar dado que tienen consecuencias que dañan y pueden ser prevenidas. Este último significado refleja claramente lo que es la ética porque permite visualizar las consecuencias de nuestros actos y nos insta a responsabilizarnos por ellos.

De ahí que se parte de la premisa de que el cuidado es la base y el componente principal de todos los procesos pedagógicos saludables, como exigencia para una pedagogía coherente y contextualizada con las nuevas demandas de sociedades plurales y complejas. Desde esta perspectiva, es necesario recuperar el sentido de pertenencia con el universo, a partir de la razón sensible y amable. Además, es indispensable el reconocimiento del valor que tiene todo lo existente, así como su derecho de convivencia con el ser humano y la interrelación existente entre todos los elementos y seres que habitan en la Tierra. A partir de esto, es primordial asumirlos como legítimos, como parte de la comunidad de la vida y que involucremos el contacto para "el reconocimiento y comunión con todos los hermanos y hermanas que nos rodean” (Boff, 2012a, p. 150).

Sumado a lo anterior, es fundamental también promover el desarrollo de una espiritualidad cósmica que nos lleve a vivir el sentido y la sensibilidad ante 
los mensajes de belleza, de grandeza y de generosidad que nos llegan de todas partes. Las cosas no son mudas. También hablan, y podemos entender la voz de los bosques y de la selva, el mensaje de los pájaros, el silbar del viento, el murmullo de los árboles, el susurro de las aguas, la mirada suplicante del pobre y el gesto afectuoso del amigo. Activar todos nuestros sentidos corporales y entrar en comunión con todas las cosas es despertar también todos los sentidos espirituales que, como las fuentes de aguas cristalinas, nos alimentan humanamente, nos producen paz, nos traen bellas inspiraciones y nos suscitan sueños bondadosos. (Boff, 2012a, pp. 150-151)

Todo lo mencionado, es un camino que posibilita el cultivo y la vivencia de una ética del cuidado, siendo esta la que provoca seres verdaderamente éticos que asumen la responsabilidad de educar y "heredar un mundo que merezca la pena vivir, trabajar, alegrarse y pasar por él, en este corto espacio de tiempo que el universo y Dios nos concedieron" (Boff, 2012a, p. 151).

Corresponde a los seres humanos, por tanto, vivir y ser portadores de la ética del cuidado a partir de la responsabilidad de perpetuar la Tierra y asegurar que esta herencia llegue hasta las generaciones futuras.

Desde esta perspectiva, es que consideramos que, dialogar sobre Pedagogía Saludable, implica abordar la perspectiva del cuidado y ubicarla en el centro de la acción educativa. La pedagogía es una trama de interdependencias que forman parte de la vida en comunidad aprendiente. Surge ahí la ética del cuidado como imperativo de las relaciones de cooperación y responsabilidad compartida; no solo como una actitud, sino como una forma de ser y hacer a favor de sí mismo, de sí misma, de las otras y de los otros. El cuidado, indica Boff (2006), "es esa condición a priori que permite que un ser conciente, racional y libre pueda existir. Sólo con cuidado, en el acto de vivir, puede éste moldear su existencia en el mundo junto con los demás hacia el futuro" (p. 45).

La pedagogía saludable propone, como ya hemos mencionado, un vivir en armonía con la persona misma y con todos los seres vivos, promoviendo el cuidado como elemento que enlaza las relaciones a partir de la espléndida perspectiva de la diversidad. Fernández y López (2010), expresan que: "Esta comunión con la comunidad de la vida es la que hemos considerado como experiencia central de la ética del cuidado" (p. 13).

A partir de lo anterior, agregamos lo sostenido por Boff (2012a), en el sentido de que el cuidado es un paradigma que propone nuevos modos de relación y, por tanto, de organizar las relaciones que establecemos con la vida, la sociedad y con nuestra Madre Tierra; por lo que es necesario asumir nuevas propuestas en los procesos educativos y pedagógicos en los que nos sumerjamos. A su criterio, "El éxito o el fracaso de esta nueva era pasará inevitablemente por la incorporación o no del paradigma del cuidado, que nos dirá si tendremos futuro como especie y si la civilización podrá seguir adelante en nuestro planeta (p. 135).

\section{Un modelo pedagógico comprometido con el cuidado}

La perspectiva de comunidad aprendiente, corresponde con un modelo pedagógico socio crítico, desde donde la historia; diálogo con la vida, se construyen nuevas conciencias, 
con visiones más complejas, diversas, holísticas que enaltecen la religación del Universo. Constituye una cosmovisión de la interdependencia, de respeto y dignificación por el entorno cotidiano, por la historia, la cultura, la humanidad, la biodiversidad.

La comunidad aprendiente germina y emerge como tallo tembloroso por lo que debe nutrirse con contactos amorosos para no morir. Precisa mirar donde nadie antes ha mirado, debe sentir donde nada ha sentido y vivir en la vida misma. La comunidad aprendiente es un organismo vivo...vibra. Es un organismo con disposición para aprender, con apertura hacia lo nuevo, hacia la innovación. (Flores, Flores, Jiménez, Madrigal y Perearnau, 2009, p. 39)

Un modelo pedagógico como orientador de la formación de un tipo determinado de persona y de sociedad, armoniza los planteamientos teóricos con las prácticas educativas cotidianas y promueve la vivencia de los principios que lo sustentan. Dicha vivencia se da en nichos de aprendizaje o ambientes de trabajo cuyas condiciones inciden en los procesos pedagógicos. "El ambiente de convivencia saludable y enriquecedora no debería ser abandonado a la posibilidad de su generación espontánea, ni tampoco ser considerado como fruto de la casualidad" (Díaz y Rodríguez, 2008, p. 162).

Una pedagogía saludable implica una metamorfosis en las mentalidades, en las sensibilidades y en las instituciones ante

la pérdida de la re-ligación de todo con todo (...) presente en la base de nuestras principales instituciones políticas y religiosas actuales, y muestra sus peligrosos límites en el descuido con planeta Tierra, en la falta de cuidado con la vida en todas sus formas y en el aumento de los conflictos en las relaciones sociales. (Boff, 2000, p. 67)

Precisamente el Modelo Pedagógico de la Universidad Nacional de Costa Rica (2008), promueve la construcción de relaciones horizontales entre docentes y estudiantes al reconocer que:

En la Universidad Nacional la docencia universitaria presupone: (...) Ambientes de escucha y conversación reflexiva donde el estudiante pueda aceptar o rechazar, con argumentos, lo que el docente propone y comprende, o viceversa. (...) Una vivencia ética, de responsabilidad ambiental y democrática. (Universidad Nacional, 2008, párr. 35)

Los modelos pedagógicos, como en el caso citado, orientan la labor institucional, lo cual responde asimismo a las aspiraciones que se comparten a nivel regional o mundial. En esta línea se cuenta con la Carta de la Tierra (2000), valioso instrumento para inspirar las relaciones entre los pueblos.

La Carta de la Tierra es una declaración internacional de principios éticos que promueven la construcción de una sociedad mundial pacífica, justa y sostenible. Es un documento que busca inspirar en todos los pueblos un nuevo sentido de interdependencia 
global y de responsabilidad compartida para el bienestar de la comunidad global y de las futuras generaciones. De particular importancia para este ensayo es citar uno de sus principios, referido al respeto y al cuidado de la comunidad de la vida que indica que:

2.- Cuidar la comunidad de la vida con entendimiento, compasión y amor:

a. Aceptar que el derecho a poseer, administrar y utilizar los recursos naturales conduce hacia el deber de prevenir daños ambientales y proteger los derechos de las personas.

b. Afirmar, que a mayor libertad, conocimiento y poder, se presenta una correspondiente responsabilidad por promover el bien común" (párr. 8).

A criterio de Boff (2012a), la Carta de la Tierra enuncia

las exigencias básicas que deben marcar el nuevo paradigma emergente del cuidado. Se trata efectivamente de un nuevo comienzo, de una verdadera conversión de las mentes y los corazones, exigida por nuestro destino común. De lo contrario, podríamos conocer el destino ya recorrido por los dinosaurios después de haber vivido 133 millones de años sobre nuestro planeta. (p. 49)

Suscribir el principio del cuidado de la comunidad de la vida significa, en primer lugar, la aceptación de que la vida en nuestro planeta constituye una colectividad, es decir, que dependemos unos y unas de otros y otras; que todos los seres vivos importamos. Por tanto, hacer explícito el cuidado como principio, es reconocer una responsabilidad compartida.

\section{La escucha, el diálogo y el silencio en la ética del cuidado}

La vida en comunidad se caracteriza, entre otras cosas, por "escuchar" y ello implica reconocer la legitimidad del otro o de la otra y una ética del cuidado requiere la capacidad de escuchar como condición básica de las relaciones.

Escuchar es obviamente algo que va más allá de la posibilidad auditiva de cada uno. Escuchar, (...) significa la disponibilidad permanente por parte del sujeto que escucha para la apertura al habla del otro, al gesto del otro, a las diferencias del otro. (Freire, 2008, p. 112)

En la pedagogía, la escucha es esencia misma del proceso y posibilita generar un clima propicio para el aprendizaje. Es componente sustancial de la comunicación y en este sentido, la persona escuchada se sabe atendida y valorada.

Mucho más importante que aquello que estás escuchando es el acto mismo de la escucha, el espacio de presencia consciente que surge al escuchar. Ese espacio es un campo 
de conciencia unificador en el que te encuentras con la otra persona sin las barreras separativas creadas por el pensamiento conceptual. Y la otra persona deja de ser «otra». (Tolle, 2009, p. 46)

La ética del cuidado en la pedagogía saludable, asume como procesos fundamentales la escucha y el silencio.

La importancia del silencio en el espacio de la comunicación es fundamental. Él me permite, por un lado, al escuchar el habla comunicante de alguien, como sujeto y no como objeto, procurar entrar en el movimiento interno de su pensamiento, volviéndome lenguaje; por el otro, torna posible a quien habla, realmente comprometido con comunicar y no con hacer comunicados, escuchar la indagación, la duda, la creación de quien escuchó. Fuera de eso, la comunicación perece. (Freire, 2008, p.110)

Los procesos pedagógicos se entienden desde la Pedagogía Saludable como espacios de convivencia es decir, espacios donde no solo se co-existe, sino donde prevalece el genuino interés por el bienestar de todas y todos; donde cada persona -con su realidad, su situación, su contexto y sus sueños- tiene cabida y es importante. Desde esta perspectiva, cobra relevancia la atención cuidadosa a las particularidades y necesidades tanto del grupo como de cada participante.

Presta atención a la pausa: la pausa entre dos pensamientos, al breve y silencioso espacio entre las palabras de una conversación, entre las notas de un piano o de una flauta, o al breve descanso entre la inspiración y la espiración. Cuando prestas atención a esas pausas, la conciencia de «algo» se convierte simplemente en conciencia. Surge de dentro de tí la dimensión informe de pura conciencia y remplaza la identificación con la forma. (Tolle, 2009, p. 7)

Un ambiente de escucha es también un ambiente de diálogo, lo cual significa en el ámbito de comunidades aprendientes, mucho más que la oportunidad de que se expresen opiniones; requiere la disposición de las personas participantes de emitir sus criterios y desprenderse de ellos, para que sea la reflexión conjunta la que lleve a su confirmación, negación o modificación. Requiere también la capacidad de cuestionarse las supuestas verdades con la apertura hacia lo desconocido, abrazando la incertidumbre. El diálogo, entendido de la manera descrita, abre espacio a la creación.

El diálogo hace posible, en suma, la presencia de una corriente de significado en el seno del grupo, a partir de la cual puede emerger una nueva comprensión, algo creativo que no se hallaba, en modo alguno, en el momento de partida. Y este significado compartido es el «aglutinante», el «cemento» que sostiene los vínculos entre las personas y entre las sociedades. (Bohm, 1997, p. 30)

Es así como podemos decir, que la pedagogía saludable se caracteriza por el diálogo constructor de paz y por el respeto mutuo. En ese marco se entiende que el cuidado no es una acción unilateral, sino que tiene una condición relacional. La persona cuidada debe sentirse cuidada. "Por tanto, el cuidado requiere reciprocidad, la receptividad del otro es necesaria en el comportamiento de cuidado" (Torras, 2013, p. 156). 
En esta misma línea de búsqueda de relaciones horizontales en los espacios pedagógicos y, coherente con ello, la pedagogía saludable participa del principio de cooperación y de autonomía.

El cuidado, para que sea efectivo, debe partir del punto de vista, las demandas y el contraste con los destinatarios del cuidado. No es aceptable el modelo del cuidador como un agente moral que decide autónomamente lo que tiene que hacer, pues implica un destinatario incapaz y dependiente. (Fernández y López, 2010, p. 12)

Además, estos espacios pedagógicos los visualizamos dentro de una dinámica con vida y, por tanto autoorganizada; en la que los y las participantes existen en y por el intercambio asumido a partir de una "autonomía ligada" como forma de relación vital. En este sentido, Najmanovich (2001), señala que

Se trata entonces de pasar de un único mundo compuesto por elementos y relaciones fijadas por las leyes de la lógica clásica a 'multimundos' donde 'unidades heterogéneas' y vínculos no tiene un sentido unívoco, no están completamente determinados, no existen independientemente sino que emergen y coevolucionan en una dinámica creativa: la trama de la vida. (p. 8)

\section{La vivencia del cuidado en espacios pedagógicos saludables}

La búsqueda de un tipo de vida saludable requiere vivenciar el cuidado en los diversos espacios educativos. Si bien en un proceso pedagógico las relaciones deben ser horizontales como hemos comentado, en los procesos formales cada docente tiene la responsabilidad de generar las condiciones para la vivencia de los principios del modelo pedagógico que orientan su labor. En el modelo pedagógico de la Universidad Nacional de Costa Rica (2008), lo anterior se expresa de la siguiente manera: "Los docentes son los promotores de un adecuado ambiente dentro del aula, en donde cada espacio constituye un microcosmos en el que los estudiantes comparten elementos culturales, lenguajes, códigos y saberes específicos" (Universidad Nacional, 2008, párr. 48).

Hablar de un "ambiente adecuado" implica desde nuestro punto de vista, ir más allá de la atención a las condiciones físicas de seguridad y comodidad, para incluir las condiciones emocionales que propician el aprendizaje. Cobra aquí importancia "gatillar" el placer de aprender.

Dentro de esta nueva visión, el placer constituye una condición primordial para el aprendizaje, pues el miedo y la preocupación obstaculizan los conocimientos, mientras que el placer tiene que ver con lo que se escucha, con la coordinación de coordinaciones. (...) El placer de aprender induce a entregarse a la creatividad con cada aliento, con la dulzura que da saberse viva o vivo, con la pasión que reclama cada día, porque el placer incita a recrearse en la experiencia universal de existir. (Flores, 2010, p.44 - 45) 


\section{Conclusión}

Lo expuesto en este ensayo, podría considerarse como la utopía del cuidado, como ya lo ha mencionado Boff (2012a): la aspiración de una pedagogía saludable va dirigida a que el cuidado se convierta en premisa inherente a los procesos pedagógicos. Las autoras proponemos en un marco de transformación renovadora: revitalizar los procesos pedagógicos en los que nos involucramos; a partir de la luz energetizante y sanadora del paradigma del cuidado.

Nos posicionamos como favorecedoras del cuidado asumido, ya no como una alternativa, sino una exigencia cotidiana orientada por el camino del compromiso con la vida, en la vida y para la vida. Una relación amorosa con todo lo existente, teñida de compasión, ética y responsabilidad.

Proponemos, una pedagogía saludable cimentada en el diálogo creativo, respetuoso, recíproco y receptivo, pero también en los silencios conscientes que permiten y potencian la escucha que legitima y congrega; trascendiendo así la simple unión de los conceptos de pedagogía y salud e integrándolos desde una perspectiva compleja. "La realidad no es un escenario plano, inerte, domesticado: es espolear la interrogación, la capacidad de maravilla. Es huir de las respuestas cerradas, de las etiquetas fáciles, de las recetas" (Fradera y Guaradans, 2008. p. 7).

La pedagogía saludable, a partir de todo lo expuesto, constituye un desafío ético fascinante que, desde el cultivo de la interioridad, se abre a nuevos paisajes y a la sabiduría necesaria para perseverar en el camino del Amor Universal.

\section{Referencias}

Boff, L. (2000). El cuidado esencial. Ética de lo humano, compasión por la Tierra. Madrid, España: Editorial Trotta

Boff, L. (2002). El cuidado esencial. Ética de lo humano, compasión por la Tierra. Madrid, España: Editorial Trotta.

Boff, L. (2006). La Carta de la Tierra en acción. Hacia un mundo sostenible. Amsterdam: KIT Publishers.

Boff, L. (2012a). El cuidado necesario. Madrid, España: Editorial Trotta.

Boff, L. (2012b). ¿Qué significa el cuidado?. Diario digital nuestro país. Recuperado de http:// elpais.cr/frontend/imprimir/67697

Bohm, D. (1997). Sobre el diálogo. Barcelona: Kairós.

Castillo, I., Castillo, R. Flores, L. E. y Miranda, G. (2014). Pedagogía saludable: Despertar de un nuevo nodo. Revista Electrónica Educare, 18(2), 311-320. Recuperado de http://www. revistas.una.ac.cr/index.php/EDUCARE/issue/current 
Díaz, J. M. y Rodríguez, J. M. (2008). La educación en valores como estrategia de desarrollo y consolidación de la persona moral. Estudios sobre Educación, 15,159-169.

Dossey, L. (1999). (3ª Ed.). Tiempo, espacio y medicina. Barcelona, España: Editorial Kairós.

Fernández, A. y López, M. (2010). La educación en valores desde la carta de la tierra. Por una pedagogía del cuidado. Revista Iberoamericana de Educación, 53, (4) 1-19.

Flores, L.E., Flores, G., Jiménez, R., Madrigal. J.C., Perearnau, M.A. (2009). Comunidad Aprendiente. Costa Rica. ARTEMUSA.

Flores, L.E. (2010). El placer de aprender. Revista Electrónica Educare, 14, 41-47. Recuperado de http://www.una.ac.cr/educare/vol14nextra10 el placer.html

Fradera. M y Guardans, T (2008). La séptima dirección. El cultivo de la interioridad. Barcelona: Claret.

Freire, P. (2008). Pedagogía de la autonomía. Argentina: Siglo XXI.

La Carta de la Tierra Internacional. (2000). La carta de la tierra. Recuperado de http://www. earthcharterinaction.org/contenido/pages/La-Carta-de-la-Tierra.html

Maturana, H. y Varela, F. (1986). El árbol del conocimiento. Las bases biológicas del entendimiento humano. Santiago de Chile: Editorial Universitaria.

Najmanovich, D. (2001,enero). Pasos hacia un pensamiento complejo en salud. Ponencia presentada en el Primer Seminario Bienal de Implicaciones Filosóficas de las Ciencias de la Complejidad. La Habana, Cuba. Recuperado de http://www.denisenajmanovich. com.ar/htmls/0303 textos.php

Tolle, E. (2009). El Silencio Habla. Madrid: Gaia.

Torras, M. (2013). El aprendizaje colaborativo en línea y la ética del cuidado. Estudios Sobre Educación, (24), 149-171.

Universidad Nacional.(2008). Modelopedagógico de la Universidad Nacional. Sistema de gestión y descarga de documentos electrónicos, Vicerrectoría Académica. Recuperado de http:// www.una.ac.cr/index.php?option $=$ com remository\&Itemid $=0 \&$ func $=$ startdown\&id $=141$ 